\title{
HOMOTOPY AND TOPOLOGICAL ACTIONS ON SPACES WITH FEW HOMOTOPY GROUPS
}

\author{
MICHAEL S. POSTOL \\ (Communicated by Frederick R. Cohen)
}

\begin{abstract}
Cooke [7] studied the problem of replacing homotopy actions by topological actions. In this paper, we use Cooke's results to show that this can always be done for a large class of spaces having few homotopy groups.
\end{abstract}

\section{INTRODUCTION}

The group of homeomorphisms of a space $X$ has long been studied in the context of covering spaces and Riemann surfaces. On the other hand, the group $\mathscr{E}(X)$ of homotopy classes of based homotopy self-equivalences of $X$ is a much more recent concept.

The first results on $\mathscr{E}(X)$ to appear in print were in a 1958 paper of Barcus and Barratt [4]. The discussion was an application of their results on homotopy classification. The first papers which dealt exclusively with the group of self-equivalences appeared in 1964 and were due to Kahn [11], Shih [18], and Arkowitz and Curjel $[2,3]$. The first general results relating the group $\mathscr{E}(X)$ to the group of homeomorphisms of $X$ appeared in a paper by Cooke in 1978 [7]. Cooke studied the following problem: a homotopy action of a group $G$ on a space $X$ is a homomorphism $\alpha: G \rightarrow \mathscr{E}(X)_{f}$, where $\mathscr{E}(X)_{f}$ is the group of free homotopy self-equivalences of $X$. A homotopy action of $G$ on $X$ is called a topological action if the image of $\alpha$ is a group of homeomorphisms of $X$. When is a homotopy action of $G$ on $X$ equivalent to a topological action of $G$ on a homotopy equivalent space $Y$ ?

Cooke showed that this problem has an affirmative solution if and only if we can solve a certain lifting problem involving classifying spaces of function spaces. He showed that the homotopy action of a free group on $X$ is equivalent to a topological action, and he gave an example of an action of $\mathscr{Z} / 2$ on a space $X$ which is not equivalent to a topological action.

The approach in this paper is to try to apply the results of Cooke to various specific cases. The difficulties in achieving this goal arise from the fact that very little is known about the function spaces which are involved in Cooke's lifting problem. Using the results of Yamanoshita [22, 23] and Siegel [19], however, I have obtained a great deal of information in the cases where $X$ is a simply

Received by the editors May 10, 1990.

1980 Mathematics Subject Classification (1985 Revision). Primary 55P10; Secondary 55R35. 
connected space with only one or two nontrivial homotopy groups. Applying Cooke's theorem to spaces with three or more homotopy groups would be very difficult, because the structure of the group $\mathscr{E}(X)$ and the space $G(X)$ of selfhomotopy equivalences of $X$ are not known in these cases.

In $\S 2$, we give the full statement of Cooke's theorem, and we define all of the spaces and maps which occur in his lifting problem. In $\S 3$, we show that if $X=K(\Pi, n)$ for $n>1$, and $\Pi$ is a finitely generated abelian group, then any homotopy action of $G$ on $X$ is equivalent to a topological action. In $\S 4$, we show that if $X$ is a stable 2-stage space such that the group $R$ in Shih's exact sequence for $\mathscr{E}(X)$ [18] is equal to 0 , then any homotopy action of a group $G$ on $X$ is equivalent to a topological action. Finally, $\S 5$ examines the 2 -stage approximation to Cooke's negative example. We show that any action of $\mathscr{Z} / 2$ on such a space is equivalent to a topological action. This strongly suggests that Cooke's example requires the space to have more than two homotopy groups and that it is unlikely that his example can be simplified.

\section{COOKE'S THEOREM}

A homotopy action of a group $G$ on a space $X$ is a homomorphism $\alpha$ from $G$ to the group $\mathscr{E}_{f}(X)$ of free homotopy classes of homotopy equivalences of $X$. Although Cooke's theorem holds for any space $X$ having the homotopy type of a CW-complex, I will make the additional assumption in all that follows that $X$ is 1 -connected. In this case, $\mathscr{E}_{f}(X)$ is isomorphic to the group $\mathscr{E}(X)$ of base-point preserving homotopy equivalences of $X$, and I will denote both of these groups by $\mathscr{E}(X)$. The action $\alpha$ is called topological if the image of $\alpha$ is a group of homeomorphisms. A homotopy action $\alpha$ of $G$ on $X$ is equivalent to a homotopy action $\beta$ of $G$ on $Y$ if there exists homotopy equivalence $f: X \rightarrow Y$ such that the diagram

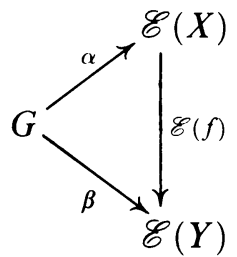

is commutative. Here $\mathscr{E}(f)$ is defined by $[g] \rightarrow\left[f g f^{-1}\right]$, where $[g] \in \mathscr{E}(X)$ and $f^{-1}$ is any homotopy inverse for $f$.

Let $G(X)$ denote the space of self-homotopy equivalences of $X$. It is an associative $H$-space satisfying $\pi_{0}(G(X))=\mathscr{E}(X)$. Let $G_{1}(X)$ be the component of the identity of $G(X)$. There is an exact sequence of $H$-spaces $G_{1}(X) \rightarrow G(X) \rightarrow \mathscr{E}(X)$ where the first map is inclusion and the second is the projection map $\rho$. The map $\rho$ induces a map $B \rho: B_{G(X)} \rightarrow K(\mathscr{E}(X), 1)$ of classifying spaces with fiber homotopy equivalent to $B_{G_{1}(X)}$.

Given a homotopy action $\alpha$, we have the following lifting problem:

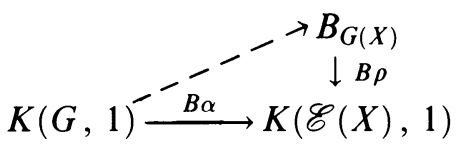

Theorem 1 (Cooke). The homotopy action $\alpha$ is equivalent to a topological action of $G$ on some space $Y$ if and only if the lifting problem above has an affirmative solution. 
Cooke gives two specific examples. First of all, he uses a telescope construction to show that $\alpha$ is equivalent to a topological action if $G$ is a free group. So if $g$ is a self-homotopy equivalence of $X$ of infinite order, $X$ can be deformed so that the image of $g$ under $\mathscr{E}(f)$ is homotopic to a homeomorphism of the new space. Cooke also shows that his theorem is nontrivial by exhibiting an action of $\mathscr{Z} / 2$ on the space $X=\left(S^{m} \cup_{2 \alpha} e^{n}\right) \vee S_{1}^{n-1} \vee S_{2}^{n-1}$ which is not equivalent to a topological action. Here $m \geq 3$ and $\alpha$ denotes an element in the 2-primary component of $\pi_{n-1}\left(S^{m}\right)$, such that the order of $\alpha$ is divisible by 4 .

\section{3. НомотоPY ACtions ON A SPACE $K(\Pi, n)$}

We now turn to the problem of whether a homotopy action of a group $G$ on a space $X$ is equivalent to a topological action when $X$ has only one or two homotopy groups. If $X$ is an Eilenberg-Mac Lane space, we will show that the homotopy action of $G$ on $X$ is equivalent to a topological action. If $X$ has two nontrivial homotopy groups, we will show that a large class of such spaces does indeed satisfy Cooke's condition.

Theorem 2. Let $X=K(\Pi, n)$, where $n>1$ and $\Pi$ is a finitely generated abelian group. Then the homotopy action of any group $G$ on $X$ is equivalent to a topological action.

Proof. Thom [21] proved that $G(X)$ has the weak homotopy type of $K(\Pi, n) \times$ Aut $\Pi$. By Proposition 2 of Yamanoshita [23], since $K(\Pi, n)$ is an $H$-space, $G(X)$ has the weak homotopy type of $K(\Pi, n) \times G_{0}(X)$, where $G_{0}(X)$ is the subspace of $G(X)$ consisting of based homotopy equivalences. A weak homotopy equivalence is given by $\phi: K(\Pi, n) \times G_{0}(X) \rightarrow G(X)$, where $\phi(x, g)(z)=$ $x \cdot g(z)$ for $x, z \in X, g \in G_{0}(X)$.

Now $\pi_{n}(G(X)) \approx \pi_{n}(K(\Pi, n)) \oplus \pi_{n}\left(G_{0}(X)\right) \approx \Pi \oplus \pi_{n}\left(G_{0}(X)\right) \approx \Pi$. So $\pi_{n}\left(G_{0}(X)\right)=0$. Since $\pi_{i}(G(X)) \approx \pi_{i}(K(\Pi, n)) \oplus \pi_{i}\left(G_{0}(X)\right)=\pi_{i}\left(G_{0}(X)\right)$ for $i \neq n$, we have that $\pi_{0}\left(G_{0}(X)\right)=$ Aut $\Pi$, and $\pi_{i}\left(G_{0}(X)\right)=0$ for $i>0$.

We define a map $\bar{\phi}: G_{0}(X) \rightarrow$ Aut $\Pi$ as follows: if $g \in G_{0}(X)$, let $\bar{\phi}(g)$ be the element of Aut $\Pi$ defined by $g \#: \pi_{n}(X) \rightarrow \pi_{n}(X) . \bar{\phi}$ is obviously multiplicative. I claim that $\bar{\phi}$ also induces a bijective correspondence between $\pi_{0}\left(G_{0}(X)\right)$ and Aut $\Pi$. Suppose $\bar{\phi}\left(g_{1}\right)=\bar{\phi}\left(g_{2}\right)$. Then $g_{1} \#=g_{2} \#$ on $\pi_{n}(X)$, and hence on all homotopy groups of $X=K(\Pi, n)$. Thus $g_{1}$ is homotopic to $g_{2}$, and they lie in the same component of $G_{0}(X)$. So $\bar{\phi}$ is one-to-one. Similarly, if $g_{1}, g_{2} \in G_{0}(X)$ are homotopic, then $g_{1} \#=g_{2} \#: \pi_{n}(X) \rightarrow \pi_{n}(X)$. So $\bar{\phi}$ is well defined. Finally, let $h \in$ Aut $\Pi$. Then there is a homotopy class of maps $f: K(\Pi, n) \rightarrow K(\Pi, n)$ such that $f \#: \Pi \rightarrow \Pi$ is equal to $h$. Since $X$ is connected, there is a $g \in G_{0}(X)$ with $g$ homotopic to $f$. Thus $\bar{\phi}$ is onto. So $\bar{\phi}$ is a weak homotopy equivalence which is multiplicative.

Since $\Pi$ is finitely generated, Kahn [12] showed that $G_{0}(X)$ is a CWcomplex. So $\bar{\phi}$ is in fact a multiplicative homotopy equivalence. So $B_{G_{0}(X)}$ is homotopy equivalent to $B_{(\text {Aut } \Pi)}=K($ Aut $\Pi, 1)$.

Now let $\rho: G(X) \rightarrow \mathscr{E}(X)$ and $\rho^{\prime}: G_{0}(X) \rightarrow \mathscr{E}(X)$ be projections. As $\mathscr{E}(X)=$ Aut $\Pi$, and since $G_{0}(X)$ is homotopy equivalent to Aut $\Pi$, we can write $\rho^{\prime}:$ Aut $\Pi \rightarrow$ Aut $\Pi$ and $\rho^{\prime}$ can be taken to be the identity. Furthermore, if $i: G_{0}(X) \rightarrow G(X)$ is inclusion, we have that $\rho \circ i=\rho^{\prime}$. By Proposition 3.2 
of Dold and Lashof [9] we have that $B \rho \circ B i=B \rho^{\prime}$. So we have the following commutative diagram for the homotopy action $\alpha: G \rightarrow \mathscr{E}(X)$ :

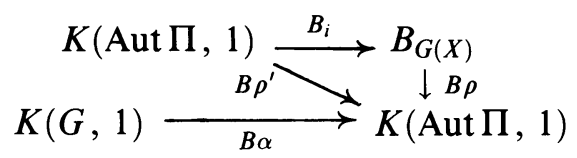

Note that $B \rho^{\prime}$ is the identity on $K($ Aut $\Pi, 1)$ since we take $\rho^{\prime}:$ Aut $\Pi \rightarrow$ Aut $\Pi$ to be the identity.

Let $\psi: K(G, 1) \rightarrow B_{G(X)}$ be defined by $\psi=B i \circ\left(B \rho^{\prime}\right)^{-1} \circ B \alpha$. Then $B \rho \circ \psi=$ $B \rho \circ B i \circ\left(B \rho^{\prime}\right)^{-1} \circ B \alpha=B \rho^{\prime} \circ\left(B \rho^{\prime}\right)^{-1} \circ B \alpha=\mathrm{id}_{K(\text { Aut }, 1)} \circ B \alpha=B \alpha$. So $\psi$ is a lift. Thus any homotopy action of a group $G$ on $X$ is equivalent to a topological action.

Note that in general, the above argument shows that if we have a lift to $B_{G_{0}(X)}$, we automatically have a lift to $B_{G(X)}$. We will use this fact in the next section.

\section{HOMOTOPY ACTIONS ON SPACES \\ WITH TWO NONTRIVIAL HOMOTOPY GROUPS}

Now consider the case of a space $X$ having a 2-stage Postnikov system.

$$
\begin{aligned}
& F=K\left(\Pi_{1}, n_{1}\right) \stackrel{i}{\rho \downarrow} X \longrightarrow \text { * } \\
& Y=K\left(\Pi_{0}, n_{0}\right) \stackrel{l}{\longrightarrow} K\left(\Pi_{1}, n_{1}+1\right)
\end{aligned}
$$

We assume that $n_{1}>n_{0}>1$ and that $\Pi_{1}, \Pi_{0}$ are finitely generated abelian groups. $l \in H^{n_{1}+1} \quad\left(K\left(\Pi_{0}, n_{0}\right) ; \Pi_{1}\right)$ is the $k$-invariant.

To compute $\mathscr{E}(X)$, we can use the following exact sequence of Shih [18] and Nomura [15]:

$$
1 \rightarrow H^{n_{1}}\left(K\left(\Pi_{0}, n_{0}\right) ; \Pi_{1}\right) \rightarrow \mathscr{E}(X) \rightarrow R \rightarrow 1 .
$$

$R$ is the subgroup of (Aut $\Pi_{0} \times$ Aut $\left.\Pi_{1}\right)$ consisting of pairs $\left(g_{0}, g_{1}\right)$ such that $g_{0} *: H^{n_{1}+1}\left(K\left(\Pi_{0}, n_{0}\right) ; \Pi_{1}\right) \rightarrow H^{n_{1}+1}\left(K\left(\Pi_{0}, n_{0}\right) ; \Pi_{1}\right)$ is the map on cohomology induced by $g_{0}, g_{1} \#$ is the coefficient automorphism induced by $g_{1}$, and $g_{0} *(l)=g_{1} \#(l)$. Nomura defines the maps in the exact sequence as follows: we have a map $\hat{\tau}: \mathscr{E}(X) \rightarrow\left(\right.$ Aut $\Pi_{0} \times$ Aut $\left.\Pi_{1}\right)$ where $[f] \in \mathscr{E}(X)$ is sent to $f_{n_{0} \#} \times f_{n_{1} \#}$ and $f_{n_{i} \#}: \pi_{n_{i}}(X) \rightarrow \pi_{n_{i}}(X)$ foi $i=0,1$. Nomura shows that $R$ is the image of $\hat{\tau}$ and that the kernel is $H^{n_{1}}\left(K\left(\Pi_{0}, n_{0}\right) ; \Pi_{1}\right)$.

Yamanoshita [22] showed that

$$
G_{0}(X) \underset{\omega}{\approx} R \times H^{n_{1}}\left(K\left(\Pi_{0}, n_{0}\right) ; \Pi_{1}\right) \times \prod_{i=1}^{n_{1}-n_{0}} K\left(H^{n_{1}-i}\left(K\left(\Pi_{0}, n_{0}\right) ; \Pi_{1}\right), i\right) .
$$

We use Yamanoshita [22] and the fact that for simply connected $X$, the group of free self-equivalences is isomorphic to the group of based self-equivalences to obtain the following result:

Proposition 3. (a) $\pi_{1}\left(B_{G_{0}(X)}\right) \approx \mathscr{E}(X)$.

(b) $\pi_{i}\left(B_{G_{0}(X)}\right) \approx H^{n_{1}-i+1}\left(K\left(\Pi_{0}, n_{0}\right) ; \Pi_{1}\right)$ for $1<i<n_{1}+1$.

(c) $\pi_{i}\left(B_{G_{0}(X)}\right)=0$ for $i \geq n_{1}+1$. 
Although we can determine the homotopy groups of $B_{G_{0}(X)}$, we do not in general know its homotopy type. The reason for this is that the weak homotopy equivalence for $G_{0}(X)$ is not usually multiplicative, so it does not uniquely determine the structure of the classifying space $B_{G_{0}(X)}$. We can, however, determine the structure of $B_{G_{0}(X)}$ when $X$ is "stable" (i.e., $n_{1} \leq 2 n_{0}-3$ ) by using the following result of Siegel [19]: Let $B_{+}(X)$ be the classifying space in the sense of Stasheff [20] and Allaud [1] for fibrations with fiber $X$, having cross sections. Then $B_{+}(X)$ is the base of a fibration with fiber $G_{0}(X)$ and contractible total space. So it is homotopy equivalent to $B_{G_{0}(X)}$, since our spaces have the homotopy type of CW-complexes [12]. We will denote both spaces by $B_{G_{0}(X)}$.

Let $K=L_{0}\left(K\left(\Pi_{0}, n_{0}\right), K\left(\Pi_{1}, n_{1}+1\right) ; 0\right)$, where $L_{0}(A, B ; 0)$ is the space of all pointed maps from $A$ to $B$ which are homotopic to a given constant map. According to Siegel, we can construct a fibration over $K$ with cross section and fiber $X$, such that the classifying map $k: K \rightarrow B_{G_{0}(X)}$ induces an isomorphism $k \#: \pi_{i}(K) \rightarrow \pi_{i}\left(B_{G_{0}(X)}\right)$ for $i>1$. (Although Siegel does not explicitly state that $K$ is a space of pointed maps, this fact is obvious, both from the context and by comparison with the results of Yamanoshita [22].)

Thom [21] observed that $K$ is a product of Eilenberg-Mac Lane spaces and proved that $\pi_{i}(K) \approx H^{n_{1}-i+1}\left(K\left(\Pi_{0}, n_{0}\right) ; \Pi_{1}\right)$, for $i \neq n_{1}+1$. So we have recovered our earlier result about the higher homotopy groups of $B_{G_{0}(X)}$. Moreover, Siegel gave the following: up to homotopy, the sequence of maps $\left.K \stackrel{k}{\rightarrow} B_{G_{0}(X)} \rightarrow K_{i}^{\prime} R, 1\right)$ is a fibration, where $R$ is the subgroup of Aut $\Pi_{0} \times$ Aut $\Pi_{1}$ in Shih's exact sequence. The action of $R$ on $\pi_{i}(K)$ is the restriction of the usual action of Aut $\Pi_{0} \times$ Aut $\Pi_{1}$ on $H^{n_{1}-i+1}\left(K\left(\Pi_{0}, n_{0}\right) ; \Pi_{1}\right)$.

Theorem 4. If $X$ is a simply connected space with a stable 2-stage Postnikov system (i.e., $n_{1} \leq 2 n_{0}-3$ ), and $R=0$, then the fibration $B \rho: B_{G_{0}(X)} \rightarrow$ $K(\mathscr{E}(X), 1)$ has a section. Hence any homotopy action of a group $G$ on $X$ is equivalent to a topological action.

Proof. If $R=0$, Siegel [19] reduces to a fibration $K \rightarrow B_{G_{0}(X)} \rightarrow *$ where $K=L_{0}\left(K\left(\Pi_{0}, n_{0}\right), K\left(\Pi_{1}, n_{1}+1\right) ; 0\right)$ as before. Since $K$ and $B_{G_{0}(X)}$ are both CW-complexes by [12], they are homotopy equivalent. Since $K$ is a product of Eilenberg-Mac Lane spaces, so is $B_{G_{0}(X)}$. Now $\mathscr{E}(X)=\pi_{1}\left(B_{G_{0}(X)}\right)$. So $K(\mathscr{E}(X), 1)$ is a factor in $B_{G_{0}(X)}$ and the map $B \rho$ is projection by the construction. So the fibration $B_{G_{0}(X)} \rightarrow K(\mathscr{E}(X), 1)$ is trivial, and it has a section.

We will now give some specific examples of stable 2-stage spaces $X$, for which $R=0$.

Proposition 5. If $\Pi_{1}=\mathscr{Z} / 2$ and $\Pi_{0}$ is cyclic, then $R=0$ if and only if $\Pi_{0}=\mathscr{Z} / 2$.

Proof. If $\Pi_{0}=\mathscr{Z} / 2$, then $R$ is contained in (Aut $\mathscr{Z} / 2 \times$ Aut $\mathscr{Z} / 2$ ) $=0$, so $R=0$.

Suppose $\Pi_{0} \neq \mathscr{Z} / 2$. Let $i$ be the generator of $H^{n_{0}}\left(K\left(\Pi_{0}, n_{0}\right) ; \mathscr{Z} / 2\right)$. Let $l \in H^{n_{1}+1}\left(K\left(\Pi_{0}, n_{0}\right) ; \mathscr{Z} / 2\right)$ be the $k$-invariant. Then by Serre [17], $l=0$ or it is a sum of elements of the form $\operatorname{Sq}^{I}(i)$. If $l=0$, then $R=\left(\right.$ Aut $\Pi_{0} \times$ 
Aut $\mathscr{Z} / 2)=$ Aut $\Pi_{0} \neq 0$. Suppose $l \neq 0$. Since $\Pi_{0} \neq \mathscr{Z} / 2$, Aut $\Pi_{0}$ contains the automorphism -1 . Now $i(-1)=1=i(1)$. So -1 fixes $i$, and $R \neq 0$.

Theorem 6. Let $\Pi_{1}=\mathscr{Z} / 2$ and $\Pi_{0}$ be a finitely generated abelian group. Then if $R=0, \Pi_{0}$ is a $\mathscr{Z} / 2$ vector space.

Proof. Let $\Pi_{0}=G_{1} \oplus \cdots \oplus G_{k}$ where the $G_{i}$ are cyclic. Then

$$
l \in H^{n_{1}+1}\left(K\left(\Pi_{0}, n_{0}\right) ; \mathscr{Z} / 2\right),
$$

which is a $\mathscr{Z} / 2$ vector space.

Suppose $G_{i} \neq \mathscr{Z} / 2$ for some $i$ with $1 \leq i \leq k$. Then let $\phi_{i}$ be the automorphism of $\Pi_{0}$ which fixes all elements of $G_{j}$ for $i \neq j$, and sends $(0, \ldots, 0,1,0, \ldots, 0) \in G_{i}$ to $(0, \ldots, 0,-1,0, \ldots, 0)$. Since any $k$-invariant $l$ is contained in a cohomology group with $\mathscr{Z} / 2$ coefficients, we have that $\phi_{i}$ fixes $l$, and $R$ cannot be 0 .

For our next theorem, we make the following comments: let $\Pi_{0}$ be a $\mathscr{Z} / 2$ vector space with $k$ summands, and $i_{1}, \ldots, i_{k}$ be the generators of $H^{n_{0}}\left(K\left(\Pi_{0}, n_{0}\right) ; \mathscr{Z} / 2\right)=\bigoplus_{i=1}^{k} H^{n_{0}}\left(K\left(\mathscr{Z} / 2, n_{0}\right) ; \mathscr{Z} / 2\right)$. Aut $\Pi_{0}$ consists of the invertible $k \times k$ matrices with coefficients in $\mathscr{Z} / 2$. Now a matrix fixes $i_{i}$ if and only if it sends the basis element $(0, \ldots, 0,1,0, \ldots, 0)$ with 1 in the $i$ th place to any element of $\Pi_{0}$ with 1 in the $i$ th place, and it sends any other basis element to an element of $\Pi_{0}$ with 0 in the $i$ th place. So the only matrix which fixes every $i_{i}$ is the identity matrix. By assumption on dimensions, the only nonzero terms in $H^{n_{1}+1}\left(K\left(\Pi_{0}, n_{0}\right) ; \mathscr{Z} / 2\right)$ are $\mathscr{Z} / 2$ vector spaces generated by elements $\operatorname{Sq}^{I}\left(i_{i}\right)$. So $R=0$ if the $k$-invariant $l$ is a sum of Steenrod squares involving every $i_{i}$.

Suppose $i_{\mid}$does not figure into $l$ for some $\mid$. Then $l$ is fixed by any invertible matrix $A$, with $A_{i, i}=1, A_{i, j}=0$ where $i \neq j, \mid$ and the $\mid$ th row is arbitrary. In order for $A$ to be invertible, there are $2^{k}-(k-1)-1$ possibilities for the $\mid$ th row of $A$. So in particular, $l$ is fixed by some matrix other than the identity, and $R \neq 0$. So we have proved the following:

Theorem 7. Let $X$ be as before. Suppose $\Pi_{1}=\mathscr{Z} / 2$ and $\Pi_{0}$ is a finitelygenerated abelian group. Then $R=0$ if and only if $\Pi_{0}=\mathscr{Z} / 2 \oplus \cdots \oplus \mathscr{Z} / 2$ and the $k$-invariant $l \in H^{n_{1}+1}\left(K\left(\Pi_{0}, n_{0}\right) ; \mathscr{Z} / 2\right)$ is a sum of Steenrod squares of all of the generators $i_{1}, \ldots, i_{k}$ of $H^{n_{0}}\left(K\left(\Pi_{0}, n_{0}\right) ; \mathscr{Z} / 2\right)$. By Cooke's theorem, any homotopy action of a group $G$ on $X$ is equivalent to a topological action.

We now examine the case where $\pi_{1} \approx \mathscr{Z} / p$ for an odd prime $p$.

Theorem 8. Let $\Pi_{0}$ be a finitely-generated abelian group, and let $\Pi_{1} \approx \mathscr{Z} / p$ for an odd prime $p$. Then $R \neq 0$.

Proof. Case $1 . \Pi_{0}$ is a $\mathscr{Z} / 2$ vector space. Then $l \in H^{n_{1}+1}\left(K\left(\Pi_{0}, n_{0}\right) ; \mathscr{Z} / p\right)=$ 0 , so $R=\left(\right.$ Aut $\Pi_{0} \times$ Aut $\left.\mathscr{Z} / p\right) \neq 0$.

Case 2. Let $\Pi_{0}=G_{1} \oplus \cdots \oplus G_{k}$ where $G_{i}$ is cyclic. Let $i_{1}, \ldots, i_{m}$ be the generators of $H^{n_{0}}\left(K\left(\Pi_{0}, n_{0}\right) ; \mathscr{Z} / p\right) \approx(\mathscr{Z} / p)^{m}$ where $G_{1}, \ldots, G_{m}$ are the summands whose orders are powers of $p$. Let $\psi \in$ Aut $\Pi_{0}$ take any basis element $(0, \ldots, 0,1,0, \ldots, 0)$ of $(\mathscr{Z} / p)^{m}$ to $(0, \ldots, 0,-1,0, \ldots, 0)$. Now $-1 \in$ Aut $\mathscr{Z} / p$, and for $1 \leq i \leq m,-1 \circ i_{i}=i_{i} \circ \psi$. Now if $l \neq 0$, then $l$ is a sum of Steenrod $p$-powers of the $i_{i}[5,6]$. So $-1_{\#}(l)=\psi^{*}(l)$ for all $i$. So $(\psi,-1) \in R$, and $R \neq 0$. 


\section{2-STAGE APPROXIMATION TO COOKE'S NEgATIVE EXAMPLE}

Recall that Cooke gives an example of a homotopy action of $\mathscr{Z} / 2$ on a space $X$ which is not equivalent to a topological action. The space $X$ is defined as follows: let $m \geq 3$ and let $\alpha$ denote any element in the 2-primary component of $\pi_{n-1}\left(S^{m}\right)$, such that the order of $\alpha$ is divisible by 4 . Then we let $X=\left(S^{m} \cup_{2 \alpha} e^{n}\right) \vee S_{1}^{n-1} \vee S_{1}^{n-1}$. Since $X$ has infinitely many nonzero homotopy groups, it is reasonable to ask whether there is a simpler example. In particular, is there an example of a homotopy action of a group $G$ on a space $X$ having only finitely many nonzero homotopy groups? The previous section shows the difficulty in answering this question, even when $X$ has only two nontrivial homotopy groups. In this section, however, we will show that for the 2-stage approximation $Y$ to one of Cooke's spaces with $n-1<2 m-1$, any homotopy action of $\mathscr{Z} / 2$ on $Y$ is equivalent to a topological action. Because of this fact, it seems unlikely that a simpler negative example can be found.

Let $X=\left(S^{m} \cup_{2 \alpha} e^{n}\right) \vee S_{1}^{n-1} \vee S_{1}^{n-1}$. In the stable range, $\pi_{m}\left(S^{m}\right) \approx \mathscr{Z}$, $\pi_{m+1}\left(S^{m}\right) \approx \mathscr{Z} / 2$. So let $Y$ be the following 2-stage space:

$$
\begin{aligned}
K(\mathscr{Z} / 2, m+1) \stackrel{i}{\longrightarrow} Y & \longrightarrow \\
& *(\mathscr{Z}, m) \stackrel{l}{\longrightarrow} K(\mathscr{Z} / 2, m+2)
\end{aligned}
$$

Theorem 9. Any homotopy action of $\mathscr{Z} / 2$ on $Y$, where $Y=K(\mathscr{Z}, m) \times$ $K(\mathscr{Z} / 2, m+1)$ or $Y$ is the 2-stage approximation to $X$ is equivalent to a topological action. (Note that in the latter case, $Y$ is the 2-stage approximation to the sphere $S^{m}$.)

Proof. The $k$-invariant $l$ is an element of $H^{m+2}(K(\mathscr{Z}, m) ; \mathscr{Z} / 2)$. So $l=0$ or $l=\mathrm{Sq}^{2}(i)$ where $i$ is the generator of $H^{m}(K(\mathscr{Z}, m) ; \mathscr{Z} / 2) \approx \mathscr{Z} / 2$.

Case 1. $l=0$. Then $Y=K(\mathscr{Z}, m) \times K(\mathscr{Z} / 2, m+1)$. According to Serre [17], $H^{m+1}(K(\mathscr{Z}, m) ; \mathscr{Z} / 2)=0$. So Shih's exact sequence gives that $\mathscr{E}(Y) \approx R \approx$ Aut $\mathscr{Z} \times$ Aut $\mathscr{Z} / 2 \approx \mathscr{Z} / 2$.

Now let $\alpha: \mathscr{Z} / 2 \rightarrow \mathscr{E}(Y)$ be a homotopy action. Let $g$ be a representative of the image of $1 \in \mathscr{Z} / 2$ under $\alpha$. Then $g_{\#}: \pi_{m}(Y) \rightarrow \pi_{m}(Y)$ is not the identity, but $g_{\#}^{2}$ is the identity. So $g_{\#}$ gives rise to a function $f: K(\mathscr{Z}, m) \rightarrow$ $K(\mathscr{Z}, m)$ such that $f$ is not homotopic to the identity, but $f^{2}$ is homotopic to the identity. So we have a homotopy action of $\mathscr{Z} / 2$ on $K(\mathscr{Z}, m)$. This action is equivalent to a topological action of $\mathscr{Z} / 2$ on a homotopy equivalent $K(\mathscr{Z}, m)$ by our earlier result. So let $\bar{f}$ be the image of $f$ under this homotopy equivalence. Then $\bar{f}$ is a homeomorphism of order 2 . So $\left(\bar{f}, \operatorname{id}_{K(\mathscr{X} / 2, m+1)}\right)$ is a homeomorphism of $Y=K(\mathscr{Z}, m) \times K(\mathscr{Z} / 2, m+1)$ of order 2 . So the action of $\mathscr{Z} / 2$ on $Y$ is equivalent to a topological action.

Case 2. $l=\mathrm{Sq}^{2}(i)$. Once again, Shih's exact sequence gives that $\mathscr{E}(Y)=R$. Now $R$ is the set of all $\phi \in$ Aut $\mathscr{Z}$ which fix $i$. But Aut $\mathscr{Z}$ consists only of the identity and multiplication by -1 . But since $i \in H^{m}(K(\mathscr{Z}, m) ; \mathscr{Z} / 2)$, $i(-1)=1=i(1)$. So any automorphism of $\mathscr{Z}$ fixes $i$, and $R=\mathscr{Z} / 2=\mathscr{E}(Y)$.

Now we claim that $Y$ is a topological group. To see this, we will first show that $Y$ is a space of loops. According to Copeland [8], $X$ has the homotopy type of a space of loops if and only if $l \in H^{m+2}(K(\mathscr{Z}, m) ; \mathscr{Z} / 2)$ is the suspension of an element $l^{\prime} \in H^{m+3}(K(\mathscr{Z}, m+1) ; \mathscr{Z} / 2)$. 
Now $l=\mathrm{Sq}^{2}(i)$ where $i$ is the generator of $H^{m}(K(\mathscr{Z}, m) ; \mathscr{Z} / 2)$. Let $l^{\prime}=\operatorname{Sq}^{2}\left(i^{\prime}\right)$ where $i^{\prime}$ is the generator of $H^{m+1}(K(\mathscr{Z}, m+1) ; \mathscr{Z} / 2)$. Let $\Sigma: H^{m+1}(K(\mathscr{Z}, m+1) ; \mathscr{Z} / 2) \rightarrow H^{m}(K(\mathscr{Z}, m) ; \mathscr{Z} / 2)$ be the suspension homomorphism. Then $\Sigma$ is an isomorphism, so $i=\Sigma i^{\prime}$. Since $\mathrm{Sq}^{2}$ commutes with suspension, we have that $l$ is the suspension of $l^{\prime}$. So $Y=\Omega Z$ where $Z$ is the following space:

$$
\begin{aligned}
K(\mathscr{Z} / 2, m+2) \underset{\rho^{\prime} \downarrow}{\longrightarrow} Z \underset{i^{\prime}}{\longrightarrow} & \downarrow \\
K(\mathscr{Z}, m+1) & \stackrel{\downarrow}{\longrightarrow} \\
K & (\mathscr{Z} / 2, m+3)
\end{aligned}
$$

Now $Z$ has the homotopy type of a countable, connected simplicial complex. So by Milnor [13], there is a universal bundle over $Z$ whose fiber is the topological group constructed as follows: henceforth, we suppose that $Z$ is a simplicial complex. Let $G$ be the space of sequences $\left[v_{0}, z_{n-1}, \ldots, z_{1}, v_{0}\right]$ topologized as a subset of $Z^{n+1}$ with $v_{0}$ a fixed vertex of $Z$ and $z_{i}$ points of $Z$ such that each pair $z_{i}, z_{i+1}$ lies in a common simplex of $Z$. Let $\bar{G}$ be the quotient space $G / \sim$ where $\left[v_{0}, z_{n-1}, \ldots, z_{i}, \ldots, z_{1}, v_{0}\right] \sim\left[v_{0}, z_{n-1}, \ldots, \hat{z}_{i}, \ldots, z_{1}, v_{0}\right]$ if $z_{i}=z_{i-1}$ or $z_{i-1}=z_{i+1}$. According to Milnor, $\bar{G}$ is a topological group which is a countable $\mathrm{CW}$-complex, and $\bar{G}$ is the fiber of a universal bundle over $Z$. Let $\psi: \bar{G} \rightarrow \Omega Z$ send $\left[v_{0}, z_{n-1}, \ldots, z_{1}, v_{0}\right]$ to the loop on $Z$ formed by taking the path on $Z$ which connects $z_{i}$ to $z_{i+1}$ by a linear path. Then since $\bar{G}$ is the fiber of a universal bundle over $Z, \psi$ induces isomorphisms on homotopy groups. Since $Z$ is a CW-complex, so is $\Omega Z$ by Milnor [14]. So $\psi$ is a homotopy equivalence. Hence, $Y$ has the homotopy type of a topological group.

Lemma 10. Let $G$ be a topological group. Then $\phi: g \rightarrow g^{-1}$ is multiplication by -1 on homotopy groups.

Proof. Let $\mu$ be the multiplication in $G$. Then $\mu\left(\phi, \operatorname{id}_{G}\right)(g)=\mu\left(g^{-1}, g\right)=1$. So $\mu\left(\phi, \mathrm{id}_{G}\right)$ is null-homotopic. Let $\zeta \in \pi_{n}(G)$, and $\left(\phi, \operatorname{id}_{G}\right)_{\sharp}(\zeta)=(\xi, \zeta) \in$ $\pi_{n}(G \times G)=\pi_{n}(G) \oplus \pi_{n}(G)$, where $\xi=\phi_{\#}(\zeta)$. Then $\mu_{\#}\left(\phi, \operatorname{id}_{G}\right)_{\#}(\zeta)=$ $\mu_{\#}(\xi, \zeta)=0 \in \pi_{n}(G)$. But we claim $\mu_{\#}(\xi, \zeta)=\xi+\zeta$. To see this, note that $\mu(g, 1)=g=\mu(1, g)$. So if $i_{1 \#}, i_{2 \#}$ are the inclusions of $\pi_{n}(G)$ into $\pi_{n}(G \times G)=\pi_{n}(G) \oplus \pi_{n}(G)$, then $\mu_{\#}(\xi, \zeta)=\mu_{\#}\left(i_{1 \#}(\xi), i_{2 \#}(\zeta)\right)=\mu_{\#}\left(i_{1 \#}(\xi), 0\right)+$ $\mu_{\#}\left(0, i_{2 \#}(\zeta)\right)=\xi+\zeta$. Thus, $\xi=\phi_{\#}(\zeta)=-\zeta$. So the lemma is proved.

Returning to Theorem 9, recall that $\pi_{m}(Y) \approx \pi_{m}(\bar{G}) \approx \mathscr{Z}$. So $\phi_{\#:}: \pi_{m}(\bar{G}) \rightarrow$ $\pi_{m}(\bar{G})$ is multiplication by -1 , which is not the identity. So $\phi$ is a homeomorphism on $\bar{G}$ of order 2 , which is not homotopic to the identity. So the homotopy action of $\mathscr{Z} / 2$ on $Y$ is equivalent to a topological action of $\mathscr{Z} / 2$ on the homotopy equivalent space $\bar{G}$, and Theorem 9 is proved.

We note that for the proof of Theorem 9 , we need that $\mathscr{E}(Y)=\mathscr{Z} / 2$, so that the inverse map is in the only class of order 2 . If $Y$ is a 2-stage space with $\pi_{m}(Y)=\mathscr{Z}, \pi_{m+r}(Y)=\mathscr{Z} / 2$, and $r>1$, then by Serre [17], $H^{m+r}(K(\mathscr{Z}, r) ; \mathscr{Z} / 2)$ is a nontrivial $\mathscr{Z} / 2$ vector space, so Shih's exact sequence gives that $\mathscr{E}(Y)$ has more than one element of order 2 and the argument of Theorem 9 fails in this case. If such a $Y$ is stable, however, the proof of Theorem 9 shows that there is a particular action of $\mathscr{Z} / 2$ on $Y$ which is equivalent to a topological action. 
To show that we cannot use the preceding argument for the spaces in Cooke's negative example, I note the following:

Theorem 11. Let $X=\left(S^{m} \cup_{2 \alpha} e^{n}\right) \vee S_{1}^{n-1} \vee S_{1}^{n-1}$ as in Cooke's negative example. Then $X$ does not have the homotopy type of an $H$-space.

Proof. According to Hopf [10], if $X$ has the homotopy type of an $H$-space, then the rational cohomology ring of $X$ is an exterior algebra on odd-dimensional generators. Now $H^{m}(X ; \mathbf{Q})=\mathbf{Q}=H^{n}(X ; \mathbf{Q}), H^{n-1}(X ; \mathbf{Q})=\mathbf{Q} \oplus \mathbf{Q}$, and all other cohomology groups in positive dimensions vanish. Since $m<n-1$, $H^{*}(X ; \mathbf{Q})$ has generators in dimensions $n-1$ and $n$. Since one of these must be even, we have a contradiction. So $X$ does not have the homotopy type of an $H$-space.

In conclusion, the author has proved some additional results relating to Cooke's problem in his thesis [16]. He shows that if $X$ is a simply connected stable 2-stage CW-complex, $B_{G_{0}(X)}$ and $B_{G(X)}$ are nilpotent spaces, and the fibration $B_{G_{0}(X)} \rightarrow K(\mathscr{E}(X), 1)$ does not have a section, then the fibration $B_{G(X)} \rightarrow K(\mathscr{E}(X), 1)$ does not have a section. In this case, the homotopy action of $\mathscr{E}(X)$ on $X$ by $\alpha: \mathscr{E}(X) \rightarrow \mathscr{E}(X)$, where $\alpha$ is the identity, is not equivalent to a topological action. He also proves results giving conditions for the action $\alpha$ of the group $N_{1} \times N_{2}$ on a space $X$ to be equivalent to a topological action when the restrictions of $\alpha$ to $N_{1}$ and $N_{2}$ are equivalent to topological actions.

\section{ACKNOWLEDGMENT}

The author would like to thank his adviser, Professor Donald Kahn, for his help and encouragement during the preparation of this work and throughout his graduate studies.

\section{REFERENCES}

1. Guy Allaud, On the classification of fibre spaces, Math. Z. 92 (1966), 110-125.

2. M. Arkowitz and C. R. Curjel, The group of homotopy equivalences, Bull. Amer. Math. Soc. 70 (1964), 293-296.

3. __ The Hurewicz homomorphism and finite homotopy invariants, Trans. Amer. Math. Soc. 110 (1964), 538-551.

4. W. D. Barcus and M. G. Barratt, On the homotopy classification of the extensions of a fixed map, Trans. Amer. Math. Soc. 88 (1958), 57-74.

5. Henri Cartan, Sur les groupes d'Eilenberg-Mac Lane $H(\Pi, n)$. I. Mèthode des constructions, Proc. Nat. Acad. Sci. U.S.A. 40 (1954), 467-471.

6. __ Sur les groupes d'Eilenberg-Mac Lane. II, Proc. Nat. Acad. Sci. U.S.A. 40 (1954), 704-707.

7. George Cooke, Replacing homotopy actions by topological actions, Trans. Amer. Math. Soc. 237 (1978), 391-406.

8. Arthur Copeland, Jr., On H-spaces with 2 nontrivial homotopy groups, Proc. Amer. Math. Soc. 8 (1957), 184-191.

9. A. Dold and R. Lashof, Principal quasi-fibrations and fibre homotopy equivalence of bundles, Illinois J. Math. 3 (1959), 285-305.

10. Heinz Hopf, Über die Topologie der Gruppen-Mannigfaltigkeiten und ihre Verallgemeinerungen, Ann. of Math. 42 (1941), 22-52.

11. Donald W. Kahn, The group of homotopy equivalences, Math. Z. 84 (1964), 1-8. 
12. Peter J. Kahn, Some function spaces of CW type, Proc. Amer. Math. Soc. 90 (1984), 599607.

13. John Milnor, Construction of universal bundles, I., Ann. of Math. 63 (1956), 272-284.

14. _, On spaces having the homotopy type of a $C W$-complex, Trans. Amer. Math. Soc. 90 (1959), 272-280.

15. Yasutoshi Nomura, Homotopy equivalences in a principal fibre space, Math. Z. 92 (1966), 380-388.

16. Michael S. Postol, Realization of homotopy equivalences by homeomorphisms, Thesis, Univ. of Minnesota, 1990.

17. J. P. Serre, Cohomologie modulo 2 des complexes d'Eilenberg-Mac Lane, Comment. Math. Helv. 27 (1953), 198-232.

18. Weishu Shih, On the group $\mathscr{E}[X]$ of homotopy equivalence maps, Bull. Amer. Math. Soc. 70 (1964), 361-365.

19. Jerrold Siegel, On the structure of $B_{\infty}(F), F$ a stable space, Pacific J. Math. 76 (1978), 491-507.

20. James Stasheff, A classification theorem for fibre spaces, Topology 2 (1963), 239-246.

21. Renè Thom, L'homologie des espaces fonctionnels, Colloque de Topologie Algebrique, Louvain, 1956.

22. Tsuneyo Yamanoshita, On the spaces of self homotopy equivalences for fibre spaces, Kodai Math. J. 10 (1987), 127-142.

23. $\ldots$, On the spaces of self homotopy equivalences of certain $C W$ complexes, J. Math. Soc. Japan 37 (1985), 455-470.

School of Mathematics, University of Minnesota, Minneapolis, Minnesota 55455 